\title{
Número de Ramsey relativo a arestas de potências de caminhos
}

\author{
Dennis Clemens ${ }^{1}$, Matthew Jenssen ${ }^{2}$, Yoshiharu Kohayakawa ${ }^{4}$, Natasha Morrison ${ }^{5}$, \\ Guilherme Oliveira Mota ${ }^{3,4}$, Damian Reding ${ }^{1}$, Barnaby Roberts ${ }^{2}$ \\ ${ }^{1}$ Technische Universität Hamburg, Institut für Mathematik \\ Hamburg, Germany \\ ${ }^{2}$ Department of Mathematics, London School of Economics \\ London, United Kingdom \\ ${ }^{3}$ Centro de Matemática, Computação e Cognição, Universidade Federal do ABC \\ Santo André, Brazil \\ ${ }^{4}$ Instituto de Matemática e Estatística, Universidade de São Paulo \\ São Paulo, Brazil \\ ${ }^{5}$ Mathematical Institute, University of Oxford \\ Oxford, United Kingdom \\ \{dennis.clemens|damian.reding\}@tuhh.de, \\ $\{m \cdot o \cdot j e n s s e n \mid b \cdot j \cdot r o b e r t s\} @ l s e \cdot a c \cdot u k$, \\ \{yoshi|mota\}@ime.usp.br, morrison@maths.ox.ac.uk
}

\begin{abstract}
Given graphs $G$ and $H$ and a positive integer $q$ we say that $G$ is $q$ Ramsey for $H$ if every $q$-colouring of the edges of $G$ contains a monochromatic copy of $H$, denoted $G \rightarrow(H)_{q}$. The size-Ramsey number $\hat{r}(H)$ of a graph $H$ is defined to be $\hat{r}(H)=\min \left\{|E(G)|: G \rightarrow(H)_{2}\right\}$. Answering a question suggested by Conlon, we prove that $\hat{r}\left(P_{n}^{k}\right)=O(n)$ for every fixed $k$, where $P_{n}^{k}$ is the kth power of the $n$-vertex path $P_{n}$, i.e., the graph with vertex set $V\left(P_{n}\right)$ and all edges $\{u, v\}$ such that the distance between $u$ and $v$ in $P_{n}$ is at most $k$.
\end{abstract}

Resumo. Dados grafos $G$ e $H$ e um inteiro positivo $q$, dizemos que $G$ é $q$ Ramsey para $H$ se toda q-coloração das arestas de $G$ contém uma cópia monocromática de $H$. Denotamos essa propriedade por $G \rightarrow(H)_{q}$. O número de Ramsey relativo a arestas $\hat{r}(H)$ de um grafo $H$ é definido como $\hat{r}(H)=\min \left\{|E(G)|: G \rightarrow(H)_{2}\right\}$. Respondendo uma pergunta sugerida por Conlon, provamos que $\hat{r}\left(P_{n}^{k}\right)=O(n)$ para todo $k$ fixo, onde $P_{n}^{k}$ é a k-ésima potência do caminho com $n$ vértices $P_{n}$, i.e., o grafo com conjunto de vértices $V\left(P_{n}\right)$ e todas as arestas $\{u, v\}$ tais que a distância entre u e vem $P_{n}$ é no máximo $k$.

O terceiro autor foi apoiado pela FAPESP (2013/03447-6, 2013/07699-0), pelo CNPq (459335/20146, 310974/2013-5) e pelo Projeto MaCLinC/USP. O quinto autor foi apoiado pela FAPESP (2013/114312, 2013/03447-6) e parcialmente pelo CNPq (459335/2014-6). A colaboração de parte dos autores foi apoiada pelo projeto CAPES/DAAD PROBRAL (430/15). Essa pesquisa foi realizada enquanto os autores participavam do ATI-HIMR Focused Research Workshop: Large-scale structures in random graphs 


\section{Introdução}

Dados grafos $G$ e $H$ e um inteiro positivo $q$, dizemos que $G$ é q-Ramsey para $H$ se toda $q$-coloração das arestas de $G$ contém uma cópia monocromática de $H$. Denotamos essa propriedade por $G \rightarrow(H)_{q}$. Quando $q=2$, escrevemos simplesmente $G \rightarrow H$. Em sua forma mais simples, o clássico Teorema de Ramsey [Ramsey 1930] afirma que para qualquer grafo $H$ existe um inteiro $N$ tal que $K_{N} \rightarrow H$. O número de Ramsey $r(H)$ de um grafo $H$ é definido como o menor tal inteiro $N$. Problemas em Teoria de Ramsey tem sido bastante estudados e muitas técnicas elegantes têm sido desenvolvidas para estimar os números de Ramsey. O trabalho de Conlon, Fox e Sudakov [Conlon et al. 2015] contém um resumo detalhado dos avanços na área.

Diversas variantes do clássico problema de Ramsey foram introduzidas e continuam a ser muito estudadas (uma boa introdução a esses problemas relacionados pode ser vista em [Conlon et al. 2015]. Em particular, Erdôs, Faudree, Rousseau e Schelp [Erdős et al. 1978] propuseram o problema de determinar a menor quantidade de arestas em um grafo $G$ tal que $G \rightarrow H$. Mais precisamente, definimos o número de Ramsey relativo a arestas de $H$, denotado por $\hat{r}(H)$, como $\hat{r}(H)=\min \{|E(G)|: G \rightarrow H\}$. Aqui estamos interessados em problemas envolvendo estimar $\hat{r}(H)$.

Para qualquer grafo $H$, temos o limitante óbvio $\hat{r}(H) \leqslant\left(\begin{array}{c}r(H) \\ 2\end{array}\right)$. Um resultado de Chvátal (veja, e.g., [Erdôs et al. 1978]) garante que esse é o valor correto para o número de Ramsey relativo a arestas de grafos completos, i.e., $\hat{r}\left(K_{n}\right)=\left(\begin{array}{c}r\left(K_{n}\right) \\ 2\end{array}\right)$.

Considerando o caminho $P_{n}$ com $n$ vértices, Erdôs [Erdôs 1981] fez a seguinte pergunta: é verdade que $\lim _{n \rightarrow \infty}\left(\hat{r}\left(P_{n}\right) / n\right)=\infty$ e $\lim _{n \rightarrow \infty}\left(\hat{r}\left(P_{n}\right) / n^{2}\right)=0$ ? Utilizando uma construção probabilística, Beck [Beck 1983] provou que o número de Ramsey relativo a arestas de caminhos é linear, i.e., $\hat{r}\left(P_{n}\right)=O(n)$. Em [Alon and Chung 1988] é fornecida uma construção explícita de um grafo $G$ com $O(n)$ arestas tal que $G \rightarrow P_{n}$. Recentemente, Dudek e Prałat [Dudek and Prałat 2015] obtiveram uma prova alternativa para esse resultado (veja também [Letzter 2016]). Mais geralmente, Friedman e Pippenger [Friedman and Pippenger 1987] provaram que número de Ramsey relativo a arestas de árvores com grau máximo limitado é linear (veja também [Dellamonica 2012, Haxell and Kohayakawa 1995, Ke 1993]) e foi mostrado em [Haxell et al. 1995] que circuitos também tem número de Ramsey relativo a arestas linear.

Respondendo uma questão colocada por Beck [Beck 1990] (negativamente), que perguntou se $\hat{r}(G)$ é linear para todos os grafos $G$ com grau máximo limitado, Rödl e Szemerédi mostraram que existe um grafo $H$ com $n$ vértices e grau máximo 3 tal que $\hat{r}(H)=\Omega\left(n \log ^{1 / 60} n\right)$. O melhor limitante superior conhecido atualmente para grafos com grau máximo limitado é provado em [Kohayakawa et al. 2011], onde é provado que para todo $\Delta$ existe uma constante $c$ tal que para qualquer grafo $H$ com $n$ vértices e grau máximo $\Delta$ temos

$$
\hat{r}(H) \leqslant c n^{2-1 / \Delta} \log ^{1 / \Delta} n .
$$

Resultados adicionais sobre o número de Ramsey relativo a arestas podem ser vistos em [Ben-Eliezer et al. 2012, Kohayakawa et al. 2016, Reimer 2002].

Dado um grafo $H$ com $n$ vértices e um inteiro $k \geqslant 2$, a $k$-ésima potência $H^{k}$ de $H$ é o grafo com conjunto de vértices $V(H)$ e todas as arestas $\{u, v\}$ tais que a distância entre $u$ e $v$ em $H$ é no máximo $k$. Respondendo uma questão sugerida por 
Conlon [Conlon 2016], provamos que o número de Ramsey de potências relativo a arestas de caminhos é linear. O seguinte teorema é o nosso resultado principal.

Teorema 1. Para todo inteiro $k \geqslant 2$, temos $\hat{r}\left(P_{n}^{k}\right)=O(n)$.

Note que dado o circuito $C_{n}$ com $n$ vértices, temos $C_{n}^{k} \subseteq P_{n}^{2 k}$. Assim, o seguinte corolário segue diretamente do Teorema 1.

Corolário 2. Para todo inteiro $k \geqslant 2$, temos $\hat{r}\left(C_{n}^{k}\right)=O(n)$.

\section{Ideia da prova do Teorema 1}

Para provar o Teorema 1 precisamos mostrar que existe um grafo $G$ com $O(n)$ arestas tal que $G \rightarrow P_{n}^{k}$. Primeiramente, mostramos que existe um grafo $H$ com quantidade linear de arestas e grau máximo limitado por uma constante tal que existe uma aresta entre quaisquer subconjuntos suficientemente grandes de $V(H)$. Seja $t$ uma constante suficientemente grande com relação a $k$. Vamos considerar o "blow-up" completo $H_{t}^{k}$ da $k$-ésima potência $H^{k}$ de $H$, i.e., o grafo obtido pela troca de cada vértice $v$ de $H^{k}$ por um grafo completo com $r\left(K_{t}\right)$ vértices, a classe $C(v)$, e pela adição de, para cada aresta $\{u, v\} \in E\left(H^{k}\right)$, todas as arestas entre $C(u)$ e $C(v)$. Note que $H_{t}^{k}$ tem uma quantidade linear de arestas.

Seja $\chi: E\left(H_{t}^{k}\right) \rightarrow\{$ vermelha, azul $\}$ uma coloração das arestas de $H_{t}^{k}$. Vamos mostrar que $H_{t}^{k}$ contém uma cópia monocromática de $P_{n}^{k}$ sob $\chi$. Como toda classe $C(v)$ de $H_{t}^{k}$ contém $r\left(K_{t}\right)$ vértices, então cada classe contém uma cópia monocromática $B(v)$ de $K_{t}$. Sem perda de generalidade, o conjunto $W:=\{v \in V(H): B(v)$ é azul $\}$ tem cardinalidade pelo menos $v(H) / 2$. Seja $F:=H[W]$ o subgrafo de $H$ induzido por $W$, e seja $F^{\prime}$ o subgrafo de $F_{t}^{k} \subseteq H_{t}^{k}$ induzido por $\bigcup_{w \in W} V(B(w))$. Dada a coloração $\chi$ acima, definimos a coloração auxiliar $\chi^{\prime}$ de $E\left(F^{k}\right)$ como segue: uma aresta $\{u, v\} \in$ $E\left(F^{k}\right)$ é colorida com a cor azul se o subgrafo bipartido $F^{\prime}[V(B(u)), V(B(v))]$ de $F^{\prime}$ naturalmente induzido pelos conjuntos $V(B(u))$ e $V(B(v))$ contém um $K_{2 k, 2 k}$ azul. Caso contrário $\{u, v\}$ é colorida com a cor vermelha.

Utilizando o Teorema 1.7 de [Pokrovskiy 2017], mostramos que toda 2-coloração de $E\left(F^{k}\right)$ contém ou uma cópia azul de $P_{n}$ ou uma cópia vermelha da $k$-ésima potência $P_{n}^{k}$ de $P_{n}$. No primeiro caso, não é difícil encontrar uma cópia azul de $P_{n}^{k}$ em $H_{t}^{k}$. O segundo caso é um pouco mais complicado, mas explorando as propriedades estruturais de $H_{t}^{k}$ é possível combinar o clássico Teorema de Kôvári-T. SósTurán [Kővári et al. 1954] com uma aplicação do lema local [Erdős and Lovász 1975, p. 616] para obter uma cópia vermelha de $P_{n}^{k}$ em $H_{t}^{k}$. Detalhes dessa prova serão dados na versão completa deste trabalho.

\section{Referências}

Alon, N. and Chung, F. R. K. (1988). Explicit construction of linear sized tolerant networks. Discrete Math., 72(1-3):15-19.

Beck, J. (1983). On size Ramsey number of paths, trees, and circuits. I. J. Graph Theory, 7(1):115-129.

Beck, J. (1990). On size Ramsey number of paths, trees and circuits. II. In Mathematics of Ramsey theory, volume 5 of Algorithms Combin., pages 34-45. Springer, Berlin. 
Ben-Eliezer, I., Krivelevich, M., and Sudakov, B. (2012). The size Ramsey number of a directed path. J. Combin. Theory Ser. B, 102(3):743-755.

Conlon, D. (2016). Problema sugerido para o ATI-HIMR Focused Research Workshop: Large-scale structures in random graphs, Alan Turing Institute, Dezembro de 2016.

Conlon, D., Fox, J., and Sudakov, B. (2015). Recent developments in graph Ramsey theory. In Surveys in combinatorics 2015, volume 424 of London Math. Soc. Lecture Note Ser., pages 49-118. Cambridge Univ. Press, Cambridge.

Dellamonica, Jr., D. (2012). The size-Ramsey number of trees. Random Structures Algorithms, 40(1):49-73.

Dudek, A. and Prałat, P. (2015). An alternative proof of the linearity of the size-Ramsey number of paths. Combin. Probab. Comput., 24(3):551-555.

Erdős, P. (1981). On the combinatorial problems which I would most like to see solved. Combinatorica, 1(1):25-42.

Erdős, P., Faudree, R. J., Rousseau, C. C., and Schelp, R. H. (1978). The size Ramsey number. Period. Math. Hungar., 9(1-2):145-161.

Erdős, P. and Lovász, L. (1975). Problems and results on 3-chromatic hypergraphs and some related questions. In Infinite and finite sets (Colloq., Keszthely, 1973; dedicated to P. Erdös on his 60th birthday), Vol. II, pages 609-627. Colloq. Math. Soc. János Bolyai, Vol. 10. North-Holland, Amsterdam.

Friedman, J. and Pippenger, N. (1987). Expanding graphs contain all small trees. Combinatorica, 7(1):71-76.

Haxell, P. E. and Kohayakawa, Y. (1995). The size-Ramsey number of trees. Israel J. Math., 89(1-3):261-274.

Haxell, P. E., Kohayakawa, Y., and Łuczak, T. (1995). The induced size-Ramsey number of cycles. Combin. Probab. Comput., 4(3):217-239.

Ke, X. (1993). The size Ramsey number of trees with bounded degree. Random Structures Algorithms, 4(1):85-97.

Kohayakawa, Y., Retter, T., and Rödl, V. (2016). The size-Ramsey number of short subdivisions of bounded degree graphs. Submitted, 2016.

Kohayakawa, Y., Rödl, V., Schacht, M., and Szemerédi, E. (2011). Sparse partition universal graphs for graphs of bounded degree. Adv. Math., 226(6):5041-5065.

Kővári, T., Sós, V. T., and Turán, P. (1954). On a problem of K. Zarankiewicz. Colloquium Math., 3:50-57.

Letzter, S. (2016). Path Ramsey number for random graphs. Combin. Probab. Comput., 25(4):612-622.

Pokrovskiy, A. (2017). Calculating Ramsey numbers by partitioning colored graphs. Journal of Graph Theory, 84(4):477-500.

Ramsey, F. P. (1930). On a problem of formal logic. Proc. London Math. Soc., S230(1):264.

Reimer, D. (2002). The Ramsey size number of dipaths. Discrete Math., 257(1):173-175. 